\title{
ABSOLUTE GAP-SHEAVES AND EXTENSIONS OF COHERENT ANALYTIC SHEAVES
}

\author{
BY \\ YUM-TONG SIU
}

Thimm introduced the concept of gap-sheaves for analytic subsheaves of finite direct sums of structure-sheaves on domains of complex number spaces (Definition 9, [13]) and proved that these gap-sheaves are coherent if the subsheaves themselves are coherent (Satz 3, [13]). This concept of gap-sheaves can be readily generalized to analytic subsheaves of arbitrary analytic sheaves on general complex spaces (Definition 1, [12]). All the gap-sheaves of coherent analytic subsheaves of arbitrary coherent analytic sheaves on general complex spaces are coherent (Theorem 3, [12]). The gap-sheaves of a given analytic subsheaf depend not only on the subsheaf itself but also on the analytic sheaf in which the given subsheaf is embedded as a subsheaf.

In this paper we introduce a new notion of gap-sheaves which we call absolute gap-sheaves (Definition 3 below). These gap-sheaves arise naturally from the problem of removing singularities of local sections of a coherent analytic sheaf. They depend only on a given analytic sheaf and neither require nor depend upon an embedding of the given sheaf as a subsheaf in another analytic sheaf. We give here a necessary and sufficient condition for the coherence of absolute gap-sheaves of coherent sheaves (Theorem 1 below). This yields some results concerning removing singularities of local sections of coherent sheaves (see Remark following Corollary 2 to Theorem 1). Then we use absolute gap-sheaves to derive a theorem (Theorem 2 below) which generalizes Serre's Theorem on the extension of torsion-free coherent analytic sheaves (Theorem 1, [11]). Finally a result on extensions of global sections of coherent analytic sheaves is derived (Theorem 4 below).

Unless specified otherwise, complex spaces are in the sense of Grauert ( $\$ 1,[5])$. If $\mathscr{S}$ is an analytic subsheaf of an analytic sheaf $\mathscr{T}$ on a complex space $(X, \mathscr{H})$, then $\mathscr{S}: \mathscr{T}$ denotes the ideal-sheaf $\mathscr{I}$ defined by $\mathscr{I}_{x}=\left\{s \in \mathscr{H}_{x} \mid s \mathscr{T}_{x} \subset \mathscr{S}_{x}\right\}$ for $x \in X . E(\mathscr{S}, \mathscr{T})$ denotes $\left\{x \in X \mid \mathscr{S}_{x} \neq \mathscr{T}_{x}\right\}$. Supp $\mathscr{T}$ denotes the support of $\mathscr{T}$. If $t \in \Gamma(X, \mathscr{T})$, then Supp $t$ denotes the support of $t$. For $x \in X, t_{x}$ denotes the germ of $t$ at $x$. By the annihilator-ideal-sheaf $\mathscr{A}$ of $\mathscr{T}$ we mean the ideal-sheaf $\mathscr{A}$ defined by $\mathscr{A}_{x}=\left\{s \in \mathscr{H}_{x} \mid s \mathscr{T}_{x}=0\right\}$ for $x \in X$. If $\theta:(X, \mathscr{H}) \rightarrow\left(X^{\prime}, \mathscr{H}^{\prime}\right)$ is a holomorphic map (i.e. a morphism of ringed spaces) from $(X, \mathscr{H})$ to another complex space $\left(X^{\prime}, \mathscr{H}^{\prime}\right)$, then $R^{0} \theta(\mathscr{T})$ denotes the zeroth direct image of $\mathscr{T}$ under $\theta$. If $f \in \Gamma(X, \mathscr{H})$ and $x \in X$, we say that $f$ vanished at $x$ if $f_{x}$ is not a unit in $\mathscr{H}_{x}$.

Received by the editors March 25, 1968. 


\section{Absolute gap-sheaves.}

Definition 1. Suppose $\mathscr{S}$ is an analytic subsheaf of an analytic sheaf $\mathscr{T}$ on a complex space $(X, \mathscr{H})$ and $\rho$ is a nonnegative integer. The $\rho$ th gap-sheaf of $\mathscr{S}$ in $\mathscr{T}$, denoted by $\mathscr{S}_{[\rho] \mathcal{F}}$, is the analytic subsheaf of $\mathscr{T}$ defined as follows: For $x \in X$, $s \in\left(\mathscr{S}_{[\rho, \mathcal{F}}\right)_{x}$ if and only if there exist an open neighborhood $U$ of $x$ in $X$, a subvariety $A$ in $U$ of dimension $\leqq \rho$, and $t \in \Gamma(U, \mathscr{T})$ such that $t_{x}=s$ and $t_{y} \in \mathscr{S}_{y}$ for $y \in U-A$.

Denote the set $\left\{x \in X \mid \mathscr{S}_{x} \neq\left(\mathscr{S}_{[\rho] \mathscr{T}}\right)_{x}\right\}$ by $E^{\rho}(\mathscr{S}, \mathscr{T})$.

REMARK. When $\mathscr{S}$ and $\mathscr{T}$ are both coherent, then $x \in E^{\rho}(\mathscr{S}, \mathscr{T})$ if and only if $\mathscr{S}_{x}$ as an $\mathscr{H}_{x}$-submodule of $\mathscr{T}_{x}$ has an associated prime ideal of dimension $\leqq \rho$ (Theorem 4, [12]). $E^{\rho}(\mathscr{S}, \mathscr{T})=\varnothing$ means that for every $x \in X \mathscr{S}_{x}$ as an $\mathscr{H}_{x}$-submodule of $\mathscr{T}_{x}$ has no associated prime ideal of dimension $\leqq \rho$.

Definition 2. Suppose $\mathscr{S}$ is an analytic subsheaf of an analytic sheaf $\mathscr{T}$ on a complex space $(X, \mathscr{H})$ and $A$ is a subvariety of $X$. Then the gap-sheaf of $\mathscr{S}$ in $\mathscr{T}$ with respect to $A$, denoted by $\mathscr{S}[A]_{\mathscr{F}}$, is defined as follows: For $x \in X, s \in\left(\mathscr{S}[A]_{\mathscr{T}}\right)_{x}$ if and only if there exist an open neighborhood $U$ of $x$ in $X$ and $t \in \Gamma(U, \mathscr{T})$ such that $t_{x}=s$ and $t_{y} \in \mathscr{S}_{y}$ for $y \in U-A$.

Proposition 1. Suppose $\mathscr{S}$ is a coherent analytic subsheaf of a coherent analytic sheaf $\mathscr{T}$ on a complex space $(X, \mathscr{H})$ and $\rho$ is a nonnegative integer. Then $\mathscr{S}_{[\rho] \mathscr{T}}$ is coherent and $E^{\rho}(\mathscr{S}, \mathscr{T})$ is a subvariety of dimension $\leqq \rho$ in $X$.

Proof. See Theorem 3 [12]. This can also be derived easily from Satz 3 [13]. Q.E.D.

Proposition 2. Suppose $\mathscr{S}$ is a coherent analytic subsheaf of a coherent analytic sheaf $\mathscr{T}$ on a complex space $(X, \mathscr{H})$ and $A$ is a subvariety of $X$. Then $\mathscr{S}[A]_{\mathscr{T}}$ is coherent.

Proof. See Theorem 1 [12]. This can also be derived easily from [13, Satz 9]. Q.E.D.

Definition 3. Suppose $\mathscr{F}$ is an analytic sheaf on a complex space $X$ and $\rho$ is a nonnegative integer. The $\rho$ th absolute gap-sheaf of $\mathscr{F}$, denoted by $\mathscr{F}^{[\rho]}$, is the analytic sheaf on $X$ defined by the following presheaf: Suppose $U \subset V$ are open subsets of $X$. Then

$$
\mathscr{F}^{[\rho]}(U)=\operatorname{ind}_{A \in \mathscr{A}(U)} \Gamma(U-A, \mathscr{F}),
$$

where $\mathfrak{A}(U)$ is the directed set of all analytic subvarieties in $U$ of dimension $\leqq \rho$ directed under inclusion. $\mathscr{F}^{[\rho]}(V) \rightarrow \mathscr{F}^{[\rho]}(U)$ is induced by restriction.

REMARKS. (i) $\mathscr{F}[\rho]=\left(\mathscr{F} / 0_{[\rho] \mathscr{F}}\right)^{[\rho]}$, where 0 is the zero-subsheaf of $\mathscr{F}$.

(ii) There is a natural sheaf-homomorphism $\mu: \mathscr{F} \rightarrow \mathscr{F}^{[\rho]}$. The kernel of $\mu$ is $0_{[\rho] \mathscr{F}}$. When $E^{\rho}(0, \mathscr{F})=\varnothing, \mu$ is injective and we can regard $\mathscr{F}$ as a subsheaf of $\mathscr{F}^{[\rho]}$. In this case we denote the set $\left\{x \in X \mid \mathscr{F}_{x} \neq\left(\mathscr{F}^{[\rho]}\right)_{x}\right\}$ by $E^{\rho}(\mathscr{F})$. 
LEMma 1. Suppose $\mathscr{F}$ is a coherent analytic sheaf on a reduced complex space $(X, \mathcal{O})$ of pure dimension $n$. Suppose $0 \leqq \rho \leqq n-2$. If $E^{n-1}(0, \mathscr{F})=\varnothing$, then $\mathscr{F}^{[\rho]}$ is coherent and $E^{\rho}(\mathscr{F})$ is a subvariety of dimension $\leqq \rho$.

Proof. Let $\pi:(\tilde{X}, \tilde{\mathcal{O}}) \rightarrow(X, \mathcal{O})$ be the normalization of $(X, \mathcal{O})$. Let $\tilde{\mathscr{F}}$ be the inverse image of $\mathscr{F}$ under $\pi$ (Definition 8, [6]). Let $\mathscr{T}$ be the torsion-subsheaf of $\tilde{F}$ and $\mathscr{G}=\tilde{\mathscr{F}} \mid \mathscr{T}$. Let $Y=\operatorname{Supp} \mathscr{T} . \mathscr{T}$ and $\mathscr{G}$ are both coherent and $\mathscr{G}$ is torsion-free (Proposition 6, [1]). $\operatorname{dim} Y \leqq n-1$ (Proposition 7, [1]). We claim that

$\mathscr{G}^{[\rho]}$ is coherent and $E^{\rho}(\mathscr{G})$ is a subvariety of dimension $\leqq \rho$ in $\tilde{X}$.

Take $x \in \tilde{X}$. On some open neighborhood $U$ of $x$ in $\tilde{X} \mathscr{G}$ can be regarded as a coherent subsheaf of $\tilde{\mathcal{O}}^{p}$ for some $p$ (Proposition 9, [1]). It is clear that $\mathscr{G}^{[\rho]}$ is isomorphic to $\mathscr{G}_{[\rho] \tilde{O}^{p}}$ on $U$ and $E^{\rho}\left(\mathscr{G}, \tilde{\mathcal{O}}^{p}\right) \cap U=E^{\rho}(\mathscr{G}) \cap U$. (1) follows from Proposition 1.

Let $\mathscr{F}^{*}=R^{0} \pi(\tilde{F}), \mathscr{G}^{*}=R^{0} \pi(\mathscr{G})$, and $\left(\mathscr{G}^{[\rho]}\right)^{*}=R^{0} \pi\left(\mathscr{G}^{[\rho]}\right)$. Let $\alpha: \mathscr{F}^{*} \rightarrow \mathscr{G}^{*}$ and $\beta: \mathscr{G}^{*} \rightarrow\left(\mathscr{G}^{[\rho]}\right)^{*}$ be induced respectively by the quotient map $\tilde{F} \rightarrow \mathscr{G}$ and the inclusion map $\mathscr{G} \rightarrow \mathscr{G}^{[\rho]}$. We have a natural sheaf-homomorphism $\lambda: \mathscr{F} \rightarrow \mathscr{F} *$ (Satz 7(b), [6]). Let $Z$ be the set of all singular points of $X$. Let $\mathscr{K}$ be the kernel of $\alpha \lambda$. Then Supp $\mathscr{K} \subset Z \cup \pi(Y)$. Since $E^{n-1}(0, \mathscr{F})=\varnothing$ and $\operatorname{dim} \operatorname{Supp} \mathscr{K} \leqq n-1$, $\mathscr{K}=0 . \gamma=\beta \alpha \lambda: \mathscr{F} \rightarrow\left(\mathscr{G}^{[\rho]}\right)^{*}$ is injective. It is easily seen that $\left(\left(\mathscr{G}^{[\rho]}\right)^{*}\right)^{[\rho]}=\left(\mathscr{G}^{[\rho]}\right)^{*}$. $\gamma$ induces a sheaf-monomorphism $\gamma_{1}: \mathscr{F}^{[\rho]} \rightarrow\left(\mathscr{G}^{[\rho]}\right)^{*} . \mathscr{F}^{[\rho]} \approx \gamma_{1}\left(\mathscr{F}^{[\rho]}\right)=\gamma(\mathscr{F})_{[\rho]\left(\mathscr{G}^{[\rho]}\right)^{*}}$

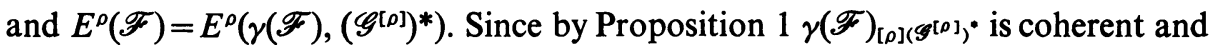
$E^{\rho}\left(\gamma(\mathscr{F}),\left(\mathscr{G}^{[\rho]}\right)^{*}\right)$ is a subvariety of dimension $\leqq \rho$ in $X$, the Lemma follows. Q.E.D.

LEMMA 2. Suppose $\mathscr{F}$ is a coherent analytic sheaf on a complex space $(X, \mathscr{H})$. Suppose $x \in X$ and $f \in \mathscr{H}_{x}$ such that for every nonnegative integer $\rho$ either $x \notin E^{\rho}(0, \mathscr{F})$ or $f$ does not vanish identically on any branch-germ of $E^{\rho}(0, \mathscr{F})$ at $x$. Then $f$ is not a zero-divisor for $\mathscr{F}_{x}$.

Proof. Suppose the contrary. Then there exist $s \in \Gamma(U, \mathscr{F})$ and $g \in \Gamma(U, \mathscr{H})$ for some open neighborhood $U$ of $x$ such that $g_{x}=f, g s=0$, and $s_{x} \neq 0$. Let $Z$ $=\operatorname{Supp} s$ and $\operatorname{dim} Z_{x}=\rho$. By shrinking $U$, we can assume that $\operatorname{dim} Z=\rho$. Hence $Z \subset E^{\rho}(0, \mathscr{F})$. Since $\operatorname{dim} E^{\rho}(0, \mathscr{F}) \leqq \rho$, the union $Z_{0}$ of all $\rho$-dimensional branches of $Z$ is equal to the union of some $\rho$-dimensional branches of $E^{\rho}(0, \mathscr{F}) \cap U$. By assumption $g$ does not vanish identically on $Z_{0}$. For some $y \in Z_{0}, g_{y}$ is a unit in $\mathscr{H}_{y} . s_{y}=0$, contradicting that $Z=\operatorname{Supp} s$. Q.E.D.

LEMMA 3. Suppose $\mathscr{F}$ is a coherent analytic sheaf on a complex space $X$ and $\rho$ is a nonnegative integer. If $E^{\rho}(0, \mathscr{F})=\varnothing$, then for any nonnegative integer $\sigma$ either $E^{\sigma}(0, \mathscr{F})=\varnothing$ or every branch of $E^{\sigma}(0, \mathscr{F})$ has dimension $>\rho$.

Proof. Suppose $Y$ is a nonempty $m$-dimensional branch of $E^{\sigma}(0, \mathscr{F})$ for some nonnegative integer $\sigma$ such that $m \leqq \rho$. Take a Stein open subset $U$ of $X$ such that $U \cap E^{\sigma}(0, \mathscr{F})=U \cap Y \neq \varnothing$. Take $x \in U \cap Y$. Since $\left(0_{[\sigma]} \mathscr{F}\right)_{x} \neq 0$, there exists 
$s \in \Gamma\left(U, 0_{[\sigma] \mathscr{F}}\right)$ such that $s_{x} \neq 0 . \operatorname{Supp} s \subset E^{\sigma}(0, \mathscr{F}) \cap U=U \cap Y . \operatorname{dim} \operatorname{Supp} s \leqq \rho$. Hence $s \in \Gamma\left(U, 0_{[\rho] \mathscr{F}}\right)$. $x \in E^{\rho}(0, \mathscr{F})$, contradicting that $E^{\rho}(0, \mathscr{F})=\varnothing$. Q.E.D.

LEMMA 4. Suppose $\mathscr{F}_{i}, 1 \leqq i \leqq 3$, are coherent analytic sheaves on a complex space $(X, \mathscr{H})$ and $\rho$ is a nonnegative integer such that $E^{\rho}\left(0, \mathscr{F}_{i}\right)=0$ for $1 \leqq i \leqq 3$. Suppose $0 \rightarrow \mathscr{F}_{1} \rightarrow \mathscr{F}_{2} \stackrel{n}{\rightarrow} \mathscr{F}_{3} \rightarrow 0$ is an exact sequence of sheaf-homomorphisms. If $\left(\mathscr{F}_{i}\right)^{[\rho]}$ is coherent and $E^{\rho}\left(\mathscr{F}_{i}\right)$ is a subvariety of dimension $\leqq \rho$ for $i=1,3$, then $\left(\mathscr{F}_{2}\right)^{[\rho]}$ is coherent and $E^{\rho}\left(\mathscr{F}_{2}\right)$ is a subvariety of dimension $\leqq \rho$.

Proof. Let $X_{i}=E^{\rho}\left(\mathscr{F}_{i}\right), i=1,3$. The problem is local in nature. Take $x_{0} \in X$ and take an open Stein neighborhood $U$ of $x_{0}$ in $X . \mathscr{F}_{i}$ is a coherent analytic subsheaf of $\left(\mathscr{F}_{i}\right)^{[\rho]}, i=1,3$. Let $\mathscr{A}_{i}=\mathscr{F}_{i}:\left(\mathscr{F}_{i}\right)^{[\rho]}, i=1,3 . E\left(\mathscr{A}_{i}, \mathscr{H}\right)=X_{i}, i=1,3$. Let $\mathscr{I}_{i}$ be the ideal-sheaf for $X_{i}, i=1,3$. By Hilbert Nullstellensatz, after shrinking $U$, we can find a natural number $m$ such that $\mathscr{I}_{i}^{m} \subset \mathscr{A}_{i}$ on $U, i=1,3$. By Lemma 3 for any nonnegative integer $\sigma$ every nonempty branch of $E^{\sigma}\left(0, \mathscr{F}_{2}\right)$ has dimension $>\rho$. Since $\operatorname{dim} X_{i} \leqq \rho, i=1,3$, we can choose $f \in \Gamma\left(U, \mathscr{I}_{1}^{m} \cap \mathscr{I}_{2}^{m}\right)$ such that $f_{x_{0}}$ does not vanish identically on any nonempty branch-germ of $E^{\sigma}\left(0, \mathscr{F}_{2}\right)$ at $x_{0}$ for any nonnegative integer $\sigma$. By Lemma $2 f_{x_{0}}$ is not a zero-divisor for $\left(\mathscr{F}_{2}\right)_{x_{0}}$. Let $\mathscr{K}$ be the kernel of the sheaf-homomorphism $\alpha: \mathscr{F}_{2} \rightarrow \mathscr{F}_{2}$ on $U$ defined by multiplication by $f$. Then $\mathscr{K}_{x_{0}}=0$. By shrinking $U$, we can assume that $\mathscr{K}=0$ on $U$. $\alpha$ induces a sheaf-monomorphism $\beta:\left(\mathscr{F}_{2}\right)^{[\rho]} \rightarrow\left(\mathscr{F}_{2}\right)^{[\rho]}$. Let $\gamma=\beta \circ \beta$. We claim that $\gamma\left(\left(\mathscr{F}_{2}\right)^{[\rho]}\right) \subset \mathscr{F}_{2}$ on $U$. Take $s \in\left(\left(\mathscr{F}_{2}\right)^{[\rho]}\right)_{x}$ for some $x \in U$. $s$ is defined by some $t \in \Gamma\left(W-A, \mathscr{F}_{2}\right)$, where $W$ is an open neighborhood of $x$ in $U$ and $A$ is a subvariety of dimension $\leqq \rho$ in $W$. $\eta(t) \in \Gamma\left(W-A, \mathscr{F}_{3}\right)$ defines an element $a$ of $\left(\left(\mathscr{F}_{3}\right)^{[\rho]}\right)_{x} . f_{x} a \in\left(\mathscr{F}_{3}\right)_{x}$. By shrinking $W$ we can find $u \in \Gamma\left(W, \mathscr{F}_{3}\right)$ such that $u$ agrees with $f \eta(t)$ on $W-A$ and we can find $v \in \Gamma\left(W, \mathscr{F}_{2}\right)$ such that $\eta(v)=u . \eta(v-f t)=0$ on $W-A . v-f t$ defines an element $b$ of $\left(\left(\mathscr{F}_{1}\right)^{[\rho]}\right)_{x} . f_{x} b \in\left(\mathscr{F}_{1}\right)_{x}$. By shrinking $W$ we can find $w \in \Gamma\left(W, \mathscr{F}_{1}\right)$ such that $w$ agrees with $f(v-f t)$ on $W-A \cdot f^{2} t=f v-w$ on $W-A \cdot \gamma(s)=\beta\left(v_{x}\right)-w_{x} \in\left(\mathscr{F}_{2}\right)_{x}$. Hence $\gamma\left(\left(\mathscr{F}_{2}\right)^{[\rho]}\right) \subset \mathscr{F}_{2}$. It is easily seen that $\gamma\left(\left(\mathscr{F}_{2}\right)^{[\rho]}\right)=\gamma\left(\mathscr{F}_{2}\right)_{[\rho] \mathscr{F}_{2}}$ on $U$ and $E^{\rho}\left(\mathscr{F}_{2}\right) \cap U$ $=E^{\rho}\left(\gamma\left(\mathscr{F}_{2}\right), \mathscr{F}_{2}\right) \cap U$. The Lemma follows from Proposition 1. Q.E.D.

LEMma 5. Suppose $\mathscr{F}$ is a coherent analytic sheaf on a complex space $(X, \mathscr{H})$ of pure dimension $n$ and $0 \leqq \rho \leqq n-2$. If $E^{n-1}(0, \mathscr{F})=\varnothing$, then $\mathscr{F}^{[\rho]}$ is coherent and $E^{\rho}(\mathscr{F})$ is a subvariety of dimension $\leqq \rho$.

Proof. Let $\mathscr{K}$ be the subsheaf of all nilpotent elements of $\mathscr{H}$ and $\mathcal{O}=\mathscr{H} \mid \mathscr{K}$. Since the lemma is local in nature, we can suppose that for some nonnegative integer $k \mathscr{K}^{k}=0$. For $0 \leqq l \leqq k$ define $\mathscr{F}^{(l)}$ inductively as follows: $\mathscr{F}^{(0)}=\mathscr{F}$ and, for $1 \leqq l \leqq k$, $\mathscr{F}^{(l)}=\left(\mathscr{K}^{F^{(l-1)}}\right)_{[n-1]} \mathscr{F}^{(l-1)}$. Let $\quad Y=\bigcup_{l=1}^{k} E^{n-1}\left(\mathscr{K}^{(l-1)}, \mathscr{F}^{(l-1)}\right) . \quad Y$ is a subvariety of dimension $\leqq n-1$. On $X-Y \mathscr{F}^{(l)}=\mathscr{K}^{\mathscr{F}^{(l-1)}}$ for $1 \leqq l \leqq k$. Hence $\mathscr{F}^{(k)}=0$ on $X-Y$. Since $\mathscr{F}^{(k)} \subset \mathscr{F}$ and $E^{n-1}(0, \mathscr{F})=\varnothing, \mathscr{F}^{(k)}=0$. From the definition of $\mathscr{F}^{(l)}$ we see that $E^{n-1}\left(\mathscr{F}^{(l)}, \mathscr{F}^{(l-1)}\right)=\varnothing$ for $1 \leqq l \leqq k$. Hence $E^{n-1}\left(0, \mathscr{F}^{(l-1)} / \mathscr{F}^{(l)}\right)$ $=\varnothing$ for $1 \leqq l \leqq k . E^{n-1}(0, \mathscr{F})=\varnothing$ implies that $E^{n-1}\left(0, \mathscr{F}^{(l)}\right)=\varnothing, 0 \leqq l \leqq k$. Since $\mathscr{K}^{(l-1)} \subset \mathscr{F}^{(l)}, \mathscr{F}^{(l-1)} / \mathscr{F}^{(l)}$ can be regarded as a coherent analytic sheaf on $(X, \mathcal{O})$, 
$1 \leqq l \leqq k$. By Lemma $1\left(\mathscr{F}^{(l-1)} / \mathscr{F}^{(l)}\right)^{[\rho]}$ is coherent and $E^{\rho}\left(\mathscr{F}^{(\rho-1)} / \mathscr{F}^{(\rho)}\right)$ is a subvariety of dimension $\leqq \rho$. Since $\mathscr{F}^{(k)}=0$, from Lemma 4 and the exact sequences $0 \rightarrow \mathscr{F}^{(l)} \rightarrow \mathscr{F}^{(l-1)} \rightarrow \mathscr{F}^{(l-1)} / \mathscr{F}^{(l)} \rightarrow 0,1 \leqq l \leqq k$, we conclude by backward induction on $l$ that $\left(\mathscr{F}^{(l)}\right)^{[\rho]}$ is coherent and $E^{\rho}\left(\mathscr{F}^{(l)}\right)$ is a subvariety of dimension $\leqq \rho$ for $0 \leqq l \leqq k$. The Lemma follows from $\mathscr{F}=\mathscr{F}(0)$. Q.E.D.

LEMMA 6. Suppose $\mathscr{F}$ is a coherent analytic sheaf on a complex space $(X, \mathscr{H})$ and $\rho$ is a nonnegative integer. Let $Y$ be the union of $(\rho+1)$-dimensional branches of $E^{\rho+1}(0, \mathscr{F})$. Then for $x \in Y\left(\mathscr{F}^{[\rho]}\right)_{x}$ is not finitely generated over $\mathscr{H}_{x}$.

Proof. We can assume that $Y \neq \varnothing$. Let $\mathscr{G}=\mathscr{F} / 0_{[\rho]} \mathscr{F}$. Since $E^{\rho}(0, \mathscr{G})=\varnothing$, by Lemma 3 and Proposition 1 every branch of $E^{\rho+1}(0, \mathscr{G})$ is $(\rho+1)$-dimensional. Since $\mathscr{G}$ agrees with $\mathscr{F}$ on $X-E^{\rho}(0, \mathscr{F}), E^{\rho+1}(0, \mathscr{G})-E^{\rho}(0, \mathscr{F})=E^{\rho+1}(0, \mathscr{F})$ $-E^{\rho}(0, \mathscr{F}) \cdot \operatorname{dim} E^{\rho}(0, \mathscr{F}) \leqq \rho$ implies that $E^{\rho+1}(0, \mathscr{G})=Y$.

Fix $x \in Y$. Suppose $\left(\mathscr{F}^{[\rho]}\right)_{x}$ is finitely generated over $\mathscr{H}_{x}$. Let $\mathscr{S}=0_{[\rho+1]} \mathscr{G}$. Since $E^{\rho}(0, \mathscr{S}) \subset E^{\rho}(0, \mathscr{G})=\varnothing, \mathscr{S} \subset \mathscr{S}^{[\rho]} \subset \mathscr{G}^{[\rho]}=\mathscr{F}[\rho]$. Since Supp $\mathscr{S}=E^{\rho+1}(0, \mathscr{G})=Y$, $\left(\mathscr{S}^{[\rho]}\right)_{x}$ is a nonzero finitely generated $\mathscr{H}_{x}$-module. Let $\left(\mathscr{S}^{[\rho]}\right)_{x}$ be generated by $s_{1}, \ldots, s_{m} \in\left(\mathscr{S}^{[\rho]}\right)_{x}$. For some open neighborhood $U$ of $x$ in $X$ and for some subvariety $A$ of dimension $\leqq \rho$ in $U s_{i}$ is induced by $t_{i} \in \Gamma(U-A, \mathscr{S}), 1 \leqq i \leqq m$. By shrinking $U$, we can choose $f \in \Gamma(U, \mathscr{H})$ such that $W=Z(f) \cap Y$ is a subvariety of dimension $\rho$ in $U$ and $x \in Z(f)$, where $Z(f)=\left\{y \in U \mid f_{y}\right.$ is not a unit in $\left.\mathscr{H}_{y}\right\}$. There exists a unique $g \in \Gamma(U-Z(f), \mathscr{H})$ such that $g f=1$ on $U-Z(f)$. For $1 \leqq i \leqq m$ define $u_{i} \in \Gamma(U-(A \cup W), \mathscr{S})$ by $\left(u_{i}\right)_{y}=0$ for $y \in U-Y$ and $\left(u_{i}\right)_{y}=\left(g t_{i}\right)_{y}$ for $y \in Y \cap(U-(A \cup W)) . u_{i}$ induces $v_{i} \in\left(\mathscr{S}^{[\rho]}\right)_{x}, 1 \leqq i \leqq m . f_{x} v_{i}=s_{i}, 1 \leqq i \leqq m$. For some $\alpha_{i j} \in \mathscr{H}_{x}, v_{i}=\sum_{j=1}^{m} \alpha_{i j} s_{j}, \quad 1 \leqq i \leqq m . \quad s_{i}=f_{x} v_{i}=\sum_{j=1}^{m} \alpha_{i j} f_{x} s_{j}, 1 \leqq i \leqq m . \quad\left(\mathscr{S}^{[\rho]}\right)_{x}$ $=f_{x}\left(\mathscr{S}^{[\rho]}\right)_{x}$. Since $f_{x}$ is not a unit in $\mathscr{H}_{x}$, by $[8,(4.1)]$ we have $\left(\mathscr{S}^{[\rho]}\right)_{x}=0$ (contradiction). Q.E.D.

THEOREM 1. Suppose $\mathscr{F}$ is a coherent analytic sheaf on a complex space $(X, \mathscr{H})$ and $\rho$ is a nonnegative integer. Then $\mathscr{F}^{[\rho]}$ is coherent if and only if $\operatorname{dim} E^{\rho+1}(0, \mathscr{F})$ $<\rho+1$. In that case $E^{\rho}\left(\mathscr{F} / 0_{[\rho\}}\right)$ is a subvariety of dimension $\leqq \rho$.

Proof. It follows from Lemma 6 that, if $\mathscr{F}^{[\rho]}$ is coherent, then $\operatorname{dim} E^{\rho+1}(0, \mathscr{F})$ $<\rho+1$.

Suppose now $\operatorname{dim} E^{\rho+1}(0, \mathscr{F})<\rho+1$. We are going to prove that $\mathscr{F}^{[\rho]}$ is coherent and $E^{\rho}\left(\mathscr{F} / 0_{[\rho]} \mathscr{F}\right)$ is a subvariety of dimension $\leqq \rho$ in $X$. Since $\mathscr{F}$ agrees with $\mathscr{F} / 0_{[\rho]} \mathscr{F}$ on $X-E^{\rho}(0, \mathscr{F}), E^{\rho+1}\left(0, \mathscr{F} / 0_{[\rho] \mathscr{F}}\right)$ is contained in the subvariety $E^{\rho}(0, \mathscr{F})$ $\cup E^{\rho+1}(0, \mathscr{F})$ of dimension $\leqq \rho . E^{\rho}\left(0, \mathscr{F} / 0_{[\rho] \mathscr{F}}\right)=\varnothing$ implies $E^{\rho+1}\left(0, \mathscr{F} / 0_{[\rho\}} \mathscr{F}\right)=\varnothing$ by Lemma 3. Since $\mathscr{F}[\rho]=\left(\mathscr{F} / 0_{[\rho] \mathscr{F}}\right)^{[\rho]}$, by replacing $\mathscr{F}$ by $\mathscr{F} / 0_{[\rho] \mathscr{F}}$, we can assume that $E^{\rho+1}(0, \mathscr{F})=\varnothing$. Since the problem is local in nature, we can suppose that $X$ is of finite dimension $n$. If $n<\rho+2, E^{\rho+1}(0, \mathscr{F})=\varnothing$ implies that $\mathscr{F}=0 . \mathscr{F}^{[\rho]}=0$ is coherent and $E^{\rho}(\mathscr{F})=\varnothing$. So we can assume that $n \geqq \rho+2$. For $\rho+1 \leqq m \leqq n$ let $\mathscr{G}^{(m)}=0_{[m] \mathscr{F}} . \quad \mathscr{G}^{(\rho+1)}=0$, because $E^{\rho+1}(0, \mathscr{F})=\varnothing$. For $\rho+2 \leqq m \leqq n$ let $X_{m}$ $=\operatorname{Supp} \mathscr{G}^{(m)} / \mathscr{G}^{(m-1)} . X_{m}$ is the union of all $m$-dimensional branches of $E^{m}(0, \mathscr{F})$, 
$\rho+2 \leqq m \leqq n . E^{m-1}\left(0, \mathscr{G}^{(m)} / \mathscr{G}^{(m-1)}\right)=\varnothing$ for $\rho+2 \leqq m \leqq n$. For $\rho+2 \leqq m \leqq n$ let $\mathscr{A}^{(m)}$ be the annihilator-ideal-sheaf for $\mathscr{G}^{(m)} / \mathscr{G}^{(m-1)}$. Then $\left(\mathscr{G}^{(m)} / \mathscr{G}^{(m-1)}\right) \mid X_{m}$ can be regarded as a coherent analytic sheaf on the complex space $\left(X_{m},\left(\mathscr{H} / \mathscr{A}^{(m)}\right) \mid X_{m}\right)$ which is either empty or of pure dimension $m, \rho+2 \leqq m \leqq n$. By Lemma 5

$$
\left(\mathscr{G}^{(m)} / \mathscr{G}^{(m-1)}\right)^{[\rho]} \approx\left(\left(\mathscr{G}^{(m)} / \mathscr{G}^{(m-1)}\right) \mid X_{m}\right)^{[\rho]}
$$

is coherent and $E^{\rho}\left(\mathscr{G}^{(m)} / \mathscr{G}^{(m-1)}\right)=E^{\rho}\left(\left(\mathscr{G}^{(m)} / \mathscr{G}^{(m-1)}\right) \mid X_{m}\right)$ is a subvariety of dimension $\leqq \rho, \rho+2 \leqq m \leqq n$. Since $\mathscr{G}^{(\rho+2)}=\mathscr{G}^{(\rho+2)} / \mathscr{G}^{(\rho+1)}$, from Lemma 4 and the exact sequences $0 \rightarrow \mathscr{G}^{(m-1)} \rightarrow \mathscr{G}^{(m)} \rightarrow \mathscr{G}^{(m)} / \mathscr{G}^{(m-1)} \rightarrow 0, \rho+3 \leqq m \leqq n$, we conclude by induction on $m$ that $\left(\mathscr{G}^{(m)}\right)^{[\rho]}$ is coherent and $E^{\rho}\left(\mathscr{G}^{(m)}\right)$ is a subvariety of dimension $\leqq \rho, \rho+2$ $\leqq m \leqq n$. The Theorem follows from $\mathscr{F}=\mathscr{G}^{(n)}$. Q.E.D.

Corollary 1. Suppose $\mathscr{F}$ is a coherent analytic sheaf on a complex space $X$, $\rho$ is a nonnegative integer, and $x \in X . \mathscr{F}^{[\rho]}$ is coherent at $x$ if and only if $x$ does not belong to a $(\rho+1)$-dimensional branch of $E^{\rho+1}(0, \mathscr{F})$. Hence the set of points where $\mathscr{F}^{[\rho]}$ is not coherent is either empty or it is a subvariety of pure dimension $\rho+1$.

REMARK. Under the assumption of Corollary 1 to Theorem $2 x$ does not belong to a $(\rho+1)$-dimensional branch of $E^{\rho+1}(0, \mathscr{F})$ if and only if the zero submodule of $\mathscr{F}_{x}$ has no associated prime ideal of dimension $\rho+1$ [12, Theorem 4]. This gives us an algebraic criterion for the coherence of $\mathscr{F}^{[\rho]}$ at $x$.

Corollary 2. Suppose $\mathscr{F}$ is a coherent analytic sheaf on a complex space $X$ and $\rho$ is a nonnegative integer. Let $\mu: \mathscr{F} \rightarrow \mathscr{F}^{[\rho]}$ be the natural sheaf-homomorphism. Then $Z=\left\{x \in X \mid \mu_{x}\right.$ is not surjective $\}$ is a subvariety of dimension $\leqq \rho+1$.

Proof. Let $Y$ be the union of all $(\rho+1)$-dimensional branches of $E^{\rho+1}(0, \mathscr{F})$. By Lemma $6 Y \subset Z$. Since $\mathscr{F}^{[\rho]}$ agrees with $\left(\mathscr{F} / 0_{\left[\rho+1 j^{\mathscr{F}}\right.}\right)^{[\rho]}$ on $X-Y, Z-Y=$ $E^{\rho}\left(\mathscr{F} / 0_{[\rho+1]^{F}}\right)-Y . Z=Y \cup E^{\rho}\left(\mathscr{F} / 0_{[\rho+1]^{F}}\right)$ is a subvariety of dimension $\leqq \rho+1$. Q.E.D.

REMARK. Corollary 2 to Theorem 1 can be stated alternatively in the following way: The set of points where we cannot always remove closed singularities contained in subvarieties of dimension $\rho$ for local sections of a coherent analytic sheaf $\mathscr{F}$ satisfying $E^{\circ}(0, \mathscr{F})=\varnothing$ is a subvariety of dimension $\leqq \rho+1$.

The weaker statement that this set of points is contained in a subvariety of dimension $\leqq \rho+1$ is an easy consequence of Satz III, [9] and Satz 5, [10].

II. Extension of coherent sheaves. Suppose $S$ is a subvariety of a complex space $X$ and $\mathscr{F}$ is a coherent analytic sheaf on $X-S . \mathscr{F}$ is said to satisfy $\left({ }^{*}\right)_{X, S}$ if for every $x \in S$ there exists some open neighborhood $U$ of $x$ in $X$ such that $\Gamma(U-S, \mathscr{F})$ generates $\mathscr{F}$ on $U-S$.

LEMMA 7. Suppose $S$ is a subvariety of codimension $\geqq 2$ in a reduced complex space $(X, \mathcal{O})$ of pure dimension $n$. Let $\theta: X-S \rightarrow X$ be the inclusion map. Suppose $\mathscr{F}$ 
is a coherent analytic sheaf on $X-S$ such that $E^{n-1}(0, \mathscr{F})=\varnothing$. If $\mathscr{F}$ satisfies $\left({ }^{*}\right)_{X, S}$, then $R^{0} \theta(\mathscr{F})$ is coherent.

Proof. Let $\pi:(\tilde{X}, \tilde{\mathcal{O}}) \rightarrow(X, \mathcal{O})$ be the normalization of $(X, \mathcal{O})$. Let $\tilde{S}=\pi^{-1}(S)$ and $\pi^{\prime}=\pi \mid(\tilde{X}-\tilde{S})$. Let $\tilde{\theta}: \tilde{X}-\tilde{S} \rightarrow \tilde{X}$ be the inclusion map. Let $\tilde{F}$ be the inverse image of $\mathscr{F}$ under $\pi^{\prime}$. Let $\mathscr{T}$ be the torsion-subsheaf of $\tilde{\mathscr{F}}, \mathscr{G}=\tilde{\mathscr{F}} \mid \mathscr{T}$, and $Y$ $=$ Supp $\mathscr{T}$. Since $\mathscr{F}$ satisfies $\left({ }^{*}\right)_{X, S}, \tilde{\mathscr{F}}$ satisfies $\left({ }^{*}\right)_{\tilde{X}, \tilde{S}}$. This implies that $\mathscr{G}$ satisfies $\left({ }^{*}\right)_{\tilde{X}, \mathcal{S}}$. By Theorem 1, [11] $R^{0} \tilde{\theta}(\mathscr{G})$ is coherent on $\tilde{X}$. Let $\mathscr{F} *=R^{0} \pi^{\prime}(\tilde{\mathscr{F}})$ and $\mathscr{G}^{*}$ $=R^{0} \pi\left(R^{0} \tilde{\theta}(\mathscr{G})\right) . \mathscr{G}^{*}$ is coherent on $X$. Let the sheaf-homomorphism $\alpha: \mathscr{F} * \rightarrow \mathscr{G}^{*}$ on $X-S$ be induced by the quotient map $\tilde{F} \rightarrow \mathscr{G}$. We have a natural sheafhomomorphism $\lambda: \mathscr{F} \rightarrow \mathscr{F *}$. Let $Z$ be the set of all singular points on $X$. Let $\mathscr{K}$ be the kernel of $\alpha \lambda$. Then Supp $\mathscr{K} \subset Z \cup \pi(Y)$. Since $E^{n-1}(0, \mathscr{F})=\varnothing$ and $\operatorname{dimSupp} \mathscr{K}$ $\leqq n-1, \mathscr{K}=0 . \alpha \lambda$ is injective. Since $R^{0} \theta\left(\mathscr{G}^{*} \mid X-S\right)=\mathscr{G}^{*}, \alpha \lambda$ induces a sheafmonomorphism $\beta: R^{0} \theta(\mathscr{F}) \rightarrow \mathscr{G}^{*}$. Take $x \in S$. There exists an open neighborhood $U$ of $x$ in $X$ such that $\Gamma(U-S, \mathscr{F})$ generates $\mathscr{F}$ on $U-S$. For $s \in \Gamma(U-S, \mathscr{F})$ let $\hat{s} \in \Gamma\left(U, \mathscr{G}^{*}\right)$ be the unique extension of $\alpha \lambda(s) .\{\hat{s} \mid s \in \Gamma(U-S, \mathscr{F})\}$ generates a coherent analytic subsheaf $\mathscr{S}$ of $\mathscr{G}^{*}$ on $U$. On $U \beta\left(R^{0} \theta(\mathscr{F})\right)=\mathscr{S}[S]_{g}$. By Proposition $2 \mathscr{S}[S]_{g} \cdot$ is coherent. Hence $R^{0} \theta(\mathscr{F})$ is coherent. Q.E.D.

Lemma 8. Suppose $S$ is a subvariety in a complex space $(X, \mathscr{H})$. Let $\theta: X-S \rightarrow X$ be the inclusion map. Suppose $\mathscr{F}_{i}, 1 \leqq i \leqq 3$, are coherent analytic sheaves on $X-S$ such that $R^{0} \theta\left(\mathscr{F}_{3}\right)$ is coherent. Suppose $0 \rightarrow \mathscr{F}_{1} \rightarrow \mathscr{F}_{2}{ }^{n} \mathscr{F}_{3} \rightarrow 0$ is an exact sequence of sheaf-homomorphisms on $X-S$. If $\mathscr{F}_{2}$ satisfies $\left({ }^{*}\right)_{X, S}$, then $\mathscr{F}_{1}$ satisfies $(*)_{X, S}$.

Proof. Take $x \in S$. There is an open neighborhood $U$ of $x$ in $X$ such that $\Gamma\left(U-S, \mathscr{F}_{2}\right)$ generates $\mathscr{F}_{2}$ on $U-S$. Let $W$ be a Stein open neighborhood of $x$ in $U$. We claim that $\Gamma\left(W-S, \mathscr{F}_{1}\right)$ generates $\mathscr{F}_{1}$ on $W-S$. Take $y \in W-S$. There exist $s_{i} \in \Gamma\left(U-S, \mathscr{F}_{2}\right), \quad 1 \leqq i \leqq m$, generating $\left(\mathscr{F}_{2}\right)_{y}$. Define a sheaf-homomorphism $\varphi: \mathscr{H}^{m} \rightarrow \mathscr{F}_{2}$ on $U-S$ by $\varphi\left(\alpha_{1}, \ldots, \alpha_{m}\right)=\sum_{i=1}^{m} \alpha_{i}\left(s_{i}\right)_{z}$ for $\alpha_{1}, \ldots, \alpha_{m} \in \mathscr{H}_{z}$ and $z \in U-S . \eta\left(s_{i}\right)$ can be extended uniquely to an element of $\Gamma\left(U, R^{0} \theta\left(\mathscr{F}_{3}\right)\right), 1 \leqq i \leqq m$. There is a unique sheaf-homomorphism $\psi: \mathscr{H}^{m} \rightarrow R^{0} \theta\left(\mathscr{F}_{3}\right)$ on $U$ which agrees with $\eta \varphi$ on $U-S$. Let $\mathscr{K}$ be the kernel of $\psi . \mathscr{K}$ is coherent. There exist $u_{i} \in \Gamma(W, \mathscr{K})$, $1 \leqq i \leqq n$, generating $\mathscr{K}_{y}$. Let $v_{i}=\varphi\left(u_{i} \mid(W-S)\right), 1 \leqq i \leqq n$. Then $v_{i} \in \Gamma\left(W-S, \mathscr{F}_{3}\right)$, $1 \leqq i \leqq n$, and $\left(\mathscr{F}_{3}\right)_{y}$ is generated by $v_{1}, \ldots, v_{n}$. Q.E.D.

Lemma 9. Suppose $S$ is a subvariety of dimension $\rho$ in a complex space $X$. Let $\theta: X-S \rightarrow X$ be the inclusion map. Suppose $\mathscr{F}_{i}, 1 \leqq i \leqq 3$, are coherent analytic sheaves on $X-S$ such that $R^{0} \theta\left(\mathscr{F}_{j}\right)$ is coherent for $j=1,3$. Suppose $0 \rightarrow \mathscr{F}_{1} \rightarrow \mathscr{F}_{2}$ $\eta_{\rightarrow} \mathscr{F}_{3} \rightarrow 0$ is an exact sequence of sheaf-homomorphisms on $X-S$. If $\mathscr{F}_{2}$ satisfies $\left({ }^{*}\right)_{X, S}$ and $E^{\rho+1}\left(0, \mathscr{F}_{2}\right)=\varnothing$, then $R^{0} \theta\left(\mathscr{F}_{2}\right)$ is coherent.

Proof. Take $x \in S$. We need only prove that $R^{0} \theta\left(\mathscr{F}_{2}\right)$ is coherent at $x$. There is a Stein open neighborhood $U$ of $x$ in $X$ such that $\Gamma\left(U-S, \mathscr{F}_{2}\right)$ generates $\mathscr{F}_{2}$ on $U-S$. 
The exact sequence $0 \rightarrow \mathscr{F}_{1} \rightarrow \mathscr{F}_{2} \stackrel{\eta}{\rightarrow} \mathscr{F}_{3} \rightarrow 0$ induces the exact sequence $0 \rightarrow$ $R^{0} \theta\left(\mathscr{F}_{1}\right) \rightarrow R^{0} \theta\left(\mathscr{F}_{2}\right) \stackrel{\eta^{\prime}}{\rightarrow} R^{0} \theta\left(\mathscr{F}_{3}\right)$. For $s \in \Gamma\left(U-S, \mathscr{F}_{2}\right)$ let $\tilde{s} \in \Gamma\left(U, R^{0} \theta\left(\mathscr{F}_{2}\right)\right)$ be the unique extension of $s$ and let $\hat{s}=\eta^{\prime}(\tilde{s})$. Let $\mathscr{S}$ be the subsheaf of $R^{0} \theta\left(\mathscr{F}_{2}\right)$ on $U$ generated by $\left\{\tilde{s} \mid s \in \Gamma\left(U-S, \mathscr{F}_{2}\right)\right\}$ and $\mathscr{T}$ be the subsheaf of $R^{0} \theta\left(\mathscr{F}_{3}\right)$ on $U$ generated by

$$
\left\{\hat{s} \mid s \in \Gamma\left(U-S, \mathscr{F}_{2}\right)\right\} .
$$

$\eta^{\prime}(\mathscr{S})=\mathscr{T}$. Since $R^{0} \theta\left(\mathscr{F}_{3}\right)$ is coherent, $\mathscr{T}$ being generated by global sections is coherent. Since $R^{0} \theta\left(\mathscr{F}_{1}\right)$ is coherent and $U$ is Stein, on $U R^{0} \theta\left(\mathscr{F}_{1}\right)$ is generated by $\Gamma\left(U, R^{0} \theta\left(\mathscr{F}_{1}\right)\right) \approx \Gamma\left(U-S, \mathscr{F}_{1}\right) \subset \Gamma\left(U-S, \mathscr{F}_{2}\right) . \quad R^{0} \theta\left(\mathscr{F}_{1}\right) \subset \mathscr{S}$. We have an exact sequence $0 \rightarrow R^{0} \theta\left(\mathscr{F}_{1}\right) \stackrel{\xi}{\rightarrow} \mathscr{S} \stackrel{\eta^{\prime \prime}}{\rightarrow} \mathscr{T} \rightarrow 0$, where $\eta^{\prime \prime}$ is induced by $\eta^{\prime}$ and $\xi$ is the inclusion map. Since $R^{0} \theta\left(\mathscr{F}_{1}\right)$ and $\mathscr{T}$ are both coherent, $\mathscr{S}$ is coherent. $E^{\rho+1}(0, \mathscr{S})$ $\subset E^{\rho+1}\left(0, \mathscr{F}_{2}\right)=\varnothing$. By Theorem $1 \mathscr{S}^{[\rho]}$ is coherent. Since $\operatorname{dim} S=\rho, R^{0} \theta\left(\mathscr{S}^{[\rho]}\right)$ $=\mathscr{S}^{[\rho]}$. The inclusion map $\mathscr{F}_{2} \rightarrow \mathscr{S}$ on $U-S$ induces on $U$ a sheaf-monomorphism $\beta: R^{0} \theta\left(\mathscr{F}_{2}\right) \rightarrow \mathscr{S}^{[\rho]} \cdot \beta\left(R^{0} \theta\left(\mathscr{F}_{2}\right)\right)=\mathscr{S}[S]_{\mathscr{S}}^{[\rho]}$. Since $\mathscr{S}[S]_{\mathscr{S}}^{[\rho]}$ is coherent by Proposition $2, R^{0} \theta\left(\mathscr{F}_{2}\right)$ is coherent on $U$. Q.E.D.

LEMMA 10. Suppose $S$ is a subvariety of codimension $\geqq 2$ in a complex space $(X, \mathscr{H})$ of pure dimension $n$. Let $\theta: X-S \rightarrow X$ be the inclusion map. Suppose $\mathscr{F}$ is a coherent analytic sheaf on $X-S$. If $\mathscr{F}$ satisfies $\left({ }^{*}\right)_{X, S}$ and $E^{n-1}(0, \mathscr{F})=\varnothing$, then $R^{0} \theta(\mathscr{F})$ is coherent on $X$.

Proof. Let $\mathscr{K}$ be the subsheaf of all nilpotent elements of $\mathscr{H}$ and $\mathcal{O}=\mathscr{H} \mid \mathscr{K}$. Since the Lemma is local in nature, we can suppose that for some nonnegative integer $k \mathscr{K}^{k}=0$. For $0 \leqq l \leqq k$ define coherent analytic sheaves $\mathscr{F}^{(l)}$ on $X-S$ inductively as follows: $\mathscr{F}^{(0)}=\mathscr{F}$ and, for $1 \leqq l \leqq k, \mathscr{F}^{(l)}=\left(\mathscr{K}^{(l-1)}\right)_{[n-1]^{(l-1)}}$. Let

$$
Y=\bigcup_{l=1}^{k} E^{n-1}\left(\mathscr{K}^{(l-1)}, \mathscr{F}^{(l-1)}\right) .
$$

$Y$ is a subvariety in $X-S$ of dimension $\leqq n-1$. On $X-(S \cup Y), \mathscr{F}^{(l)}=\mathscr{K}^{(l-1)}$ for $1 \leqq l \leqq k$. Hence $\mathscr{F}^{(k)}=0$ on $X-(S \cup Y)$. Since $\mathscr{F}^{(k)} \subset \mathscr{F}$ and $E^{n-1}(0, \mathscr{F})=\varnothing$, $\mathscr{F}^{(k)}=0$ on $X-S$. From the definition of $\mathscr{F}^{(l)}$ we see that $E^{n-1}\left(\mathscr{F}^{(l)}, \mathscr{F}^{(l-1)}\right)=\varnothing$ for $1 \leqq i \leqq k$. Hence $E^{n-1}\left(0, \mathscr{F}^{(l-1)} / \mathscr{F}^{(l)}\right)=0$ for $1 \leqq l \leqq k . E^{n-1}(0, \mathscr{F})=\varnothing$ implies that $E^{n-1}\left(0, \mathscr{F}^{(l)}\right)=\varnothing$ for $1 \leqq l \leqq k$. Since $\mathscr{H}^{(l-1)} \subset \mathscr{F}^{(l)}, \mathscr{F}^{(l-1)} / \mathscr{F}^{(l)}$ can be regarded as a coherent analytic sheaf on $(X-S, \mathcal{O} \mid(X-S)), 1 \leqq l \leqq k$.

Set $\mathscr{F}^{(k+1)}=0$. We are going to prove $(2)_{l}$ for $0 \leqq l \leqq k$ by induction on $l$ :

$$
\mathscr{F}^{(l)} \text { satisfies }\left(^{*}\right)_{X, S} \text { and } R^{0} \theta\left(\mathscr{F}^{(l)} / \mathscr{F}^{(l+1)}\right) \text { is coherent. }
$$

Since $\mathscr{F}^{(0)}=\mathscr{F}, \mathscr{F}^{(0)}$ satisfies $\left({ }^{*}\right)_{X, S} \cdot \mathscr{F}^{(0)} / \mathscr{F}^{(1)}$ satisfies $\left({ }^{*}\right)_{X, S}$. By Lemma 7

$$
R^{0} \theta\left(\mathscr{F}^{(0)} / \mathscr{F}^{(1)}\right)
$$

is coherent. (2) $)_{0}$ is true. Suppose for some $0 \leqq m<k(2)_{m}$ is true. By Lemma 8 and 
the exact sequence $0 \rightarrow \mathscr{F}^{(m+1)} \rightarrow \mathscr{F}^{(m)} \rightarrow \mathscr{F}^{(m)} / \mathscr{F}^{(m+1)} \rightarrow 0$, we conclude that $\mathscr{F}^{(m+1)}$ satisfies $\left({ }^{*}\right)_{X, S}$. Hence $\mathscr{F}^{(m+1)} / \mathscr{F}^{(m+2)}$ satisfies $\left({ }^{*}\right)_{X, S}$. By Lemma 7

$$
R^{0} \theta\left(\mathscr{F}^{(m+1)} / \mathscr{F}^{(m+2)}\right)
$$

is coherent. (2) $)_{m+1}$ is true. Hence (2) holds for $0 \leqq l \leqq k$.

Now we are going to prove (3) for $0 \leqq l \leqq k$ by backward induction on $l$ :

$$
R^{0} \theta\left(\mathscr{F}^{(l)}\right) \text { is coherent. }
$$

Since $\mathscr{F}^{(k)}=0,(3)_{k}$ is true. Suppose (3) $m$ is true for some $0<m \leqq k$. From $(2)_{m-1}$, $(3)_{m}$, Lemma 10 and the exact sequence $0 \rightarrow \mathscr{F}^{(m)} \rightarrow \mathscr{F}^{(m-1)} \rightarrow \mathscr{F}^{(m-1)} / \mathscr{F}^{(m)} \rightarrow 0$, we conclude that $(3)_{m-1}$ is true. Hence $(3)_{l}$ holds for $0 \leqq l \leqq k$. The Lemma follows from (3) $)_{0}$ Q.E.D.

Lemma 11. Suppose $S$ is a subvariety of dimension $\rho$ in a complex space $(X, \mathscr{H})$. Suppose $\mathscr{F}$ is a coherent analytic sheaf on $X-S$ such that Supp $\mathscr{F}$ is a subvariety of pure dimension $n>\rho$ and $E^{n-1}(0, \mathscr{F})=\varnothing$. Then there exists a complex subspace $(Y, \mathscr{K})$ of pure dimension $n$ in $(X, \mathscr{H})$ such that $Y-S=\operatorname{Supp} \mathscr{F}$ and $\mathscr{F} \mid(Y-S)$ can be regarded as a coherent analytic sheaf on $(Y-S, \mathscr{K} \mid(Y-S))$.

Proof. By [7, V.D.5] the topological closure $Y$ of Supp $\mathscr{F}$ in $X$ is a subvariety of pure dimension $n$. Let $Y=\bigcup_{\alpha \in A} Y_{\alpha}$ be the decomposition into irreducible branches. Let $\mathscr{I}_{\alpha}$ be the ideal-sheaf for $Y_{\alpha}, \alpha \in A$. Choose $x_{\alpha} \in Y_{\alpha}-\left(S \cup\left(\cup_{\beta \in A, \beta \neq \alpha} Y_{\beta}\right)\right)$. Let $\mathscr{A}$ be the annihilator-ideal-sheaf for $\mathscr{F}$. Then $E(\mathscr{A}, \mathscr{H} \mid(X-S))=Y-S$. By Hilbert Nullstellensatz, there exists a natural number $m_{\alpha}$ such that $\left(\mathscr{I}_{\alpha}^{m_{\alpha}}\right)_{x_{\alpha}} \subset \mathscr{A}_{x_{\alpha}}$, $\alpha \in A$. Let $\mathscr{I}=\prod_{\alpha \in A} \mathscr{I}^{m_{\alpha}}$. Then $\mathscr{I}$ is coherent and $(\mathscr{I} \mathscr{F})_{x_{\alpha}}=0$ for $\alpha \in A$. Supp $\mathscr{I} \mathscr{F}$ is a subvariety of dimension $<n$ in $X-S . E^{n-1}(0, \mathscr{F})=\varnothing$ implies that $\mathscr{I} \mathscr{F}=0$. Set $\mathscr{K}=(\mathscr{H} \mid \mathscr{I}) \mid Y$. Then $(Y, \mathscr{K})$ satisfies the requirements. Q.E.D.

TheOREM 2. Suppose $S$ is a subvariety of dimension $\rho$ in a complex space $(X, \mathscr{H})$. Let $\theta: X-S \rightarrow X$ be the inclusion map. Suppose $\mathscr{F}$ is a coherent analytic sheaf on $X-S$ such that $E^{\rho+1}(0, \mathscr{F})=\varnothing$ or equivalently for every $x \in X-S$ the zero $\mathscr{H}_{x^{-}}$ submodule of $\mathscr{F}_{x}$ has no associated prime ideal of dimension $\leqq \rho+1$. Then the following conditions are equivalent:

(i) $R^{0} \theta(\mathscr{F})$ is coherent.

(ii) There exists a coherent analytic sheaf on $X$ which extends $\mathscr{F}$.

(iii) $\mathscr{F}$ satisfies $\left({ }^{*}\right)_{X, S}$.

Proof. It is clear that (i) implies (ii) and (ii) implies (iii). We need only prove that (iii) implies (i). Suppose $\mathscr{F}$ satisfies $\left({ }^{*}\right)_{X, S}$. We are going to prove that $R^{0} \theta(\mathscr{F})$ is coherent. Since the problem is local in nature, we can suppose that $X$ is of finite dimension $n$. If $n<\rho+2$, then $E^{\rho+1}(0, \mathscr{F})=\varnothing$ implies that $\mathscr{F}=0 . R^{0} \theta(\mathscr{F})=0$ is coherent. So we can assume that $n \geqq \rho+2$. For $\rho+1 \leqq m \leqq n$ let $\mathscr{G}^{(m)}=0_{[m]} \mathscr{F}$. $\mathscr{G}^{(\rho+1)}=0$, because $E^{\rho+1}(0, \mathscr{F})=\varnothing$. For $\rho+2 \leqq m \leqq n$ let $X_{m}=\operatorname{Supp} \mathscr{G}^{(m)} / \mathscr{G}^{(m-1)}$. Then $X_{m}$ is the union of all $m$-dimensional branches of $E^{m}(0, \mathscr{F}), \rho+2 \leqq m \leqq n$. 
$E^{m-1}\left(0, \mathscr{G}^{(m)} / \mathscr{G}^{(m-1)}\right)=\varnothing$ for $\rho+2 \leqq m \leqq n$. By Lemma 11 there exists a complex subspace $\left(Y_{m}, \mathscr{K}_{m}\right)$ of pure dimension $m$ in $(X, \mathscr{H})$ such that $Y_{m}-S=X_{m}$ and $\left(\mathscr{G}^{(m)} / \mathscr{G}^{(m-1)}\right) \mid\left(Y_{m}-S\right)$ can be regarded as a coherent analytic sheaf on

$$
\left(Y_{m}-S, \mathscr{K}_{m} \mid\left(Y_{m}-S\right)\right), \rho+2 \leqq m \leqq n .
$$

Let $\theta_{m}: Y_{m}-S \rightarrow Y_{m}$ be the inclusion map $\rho+2 \leqq m \leqq n . E^{\rho+1}(0, \mathscr{F})=\varnothing$ implies that $E^{\rho+1}\left(0, \mathscr{G}^{(m)}\right)=0$ for $\rho+2 \leqq m \leqq n$.

We are going to prove (4) for $\rho+2 \leqq m \leqq n$ by backward induction on $m$ :

$$
\mathscr{G}^{(m)} \text { satisfies }\left({ }^{*}\right)_{X, S} \text { and } R^{0} \theta\left(\mathscr{G}^{(m)} / \mathscr{G}^{(m-1)}\right) \text { is coherent. }
$$

Since $\mathscr{G}^{(n)}=\mathscr{F}, \mathscr{G}^{(n)}$ satisfies $\left({ }^{*}\right)_{X, S}$. $\left(\mathscr{G}^{(n)} / \mathscr{G}^{(n-1)}\right) \mid\left(Y_{n}-S\right)$ satisfies $\left(^{*}\right)_{Y_{n}, Y_{n} \cap S}$. By Lemma $10 R^{0} \theta\left(\mathscr{G}^{(n)} / \mathscr{G}^{(n-1)}\right) \approx R^{0} \theta_{n}\left(\left(\mathscr{G}^{(n)} / \mathscr{G}^{(n-1)}\right) \mid\left(Y_{n}-S\right)\right)$ is coherent. (4) $n$ is true. Suppose for some $\rho+2<q \leqq n,(4)_{q}$ is true. From Lemma $8,(4)_{q}$, and the exact sequence $0 \rightarrow \mathscr{G}^{(q-1)} \rightarrow \mathscr{G}^{(q)} \rightarrow \mathscr{G}^{(q)} / \mathscr{G}^{(q-1)} \rightarrow 0$ we conclude that $\mathscr{G}^{(q-1)}$ satisfies $\left({ }^{*}\right)_{X, S \cdot}\left(\mathscr{G}^{(q-1)} / \mathscr{G}^{(q-2)}\right) \mid\left(Y_{q-1}-S\right)$ satisfies $\left({ }^{*}\right)_{Y_{q-1}, Y_{q-1} \cap S .}$ By Lemma $10 R^{0} \theta\left(\mathscr{G}^{(q-1)} \mid\right.$ $\left.\mathscr{G}^{(q-2)}\right) \approx R^{0} \theta_{q-1}\left(\left(\mathscr{G}^{(q-1)} / \mathscr{G}^{(q-2)}\right) \mid\left(Y_{q-1}-S\right)\right)$ is coherent. $(4)_{q-1}$ is true. Hence $(4)_{m}$ holds for $\rho+2 \leqq m \leqq n$.

Now we are going to prove $(5)_{m}$ for $\rho+1 \leqq m \leqq n$ by induction on $m$ : $R^{0} \theta\left(\mathscr{G}^{(m)}\right)$ is coherent.

Since $\mathscr{G}^{(\rho+1)}=0,(5)_{\rho+1}$ is true. Suppose $(5)_{q}$ is true for some $\rho+1 \leqq q<n$. From $(4)_{q+1},(5)_{q}$, Lemma 9 , and the exact sequence $0 \rightarrow \mathscr{G}^{(q)} \rightarrow \mathscr{G}^{(q+1)} \rightarrow \mathscr{G}^{(q+1)} / \mathscr{G}^{(q)} \rightarrow 0$ we conclude that $R^{0} \theta\left(\mathscr{G}^{(q+1)}\right)$ is coherent. $(5)_{q+1}$ is true. Hence (5) holds for $\rho+1$ $\leqq m \leqq n$. Since $\mathscr{G}^{(n)}=\mathscr{F},(5)_{n}$ implies that $R^{0} \theta(\mathscr{F})$ is coherent. Q.E.D.

CoRollary. Suppose $S$ is a subvariety of dimension $\rho$ in a complex space $(X, \mathscr{H})$ and $\theta: X-S \rightarrow X$ is the inclusion map. Suppose $\mathscr{F}$ is a coherent analytic sheaf on $X-S$ such that the homological codimension (p. 358, [9]) of the $\mathscr{H}_{x}$-module $\mathscr{F}_{x} \geqq \rho+2$ for $x \in X$. Then the following conditions are equivalent:

(i) $R^{0} \theta(\mathscr{F})$ is coherent.

(ii) There exists a coherent analytic sheaf on $X$ which extends $\mathscr{F}$.

(iii) $\mathscr{F}$ satisfies $\left({ }^{*}\right)_{X, S}$.

Proof. Follows from Theorem 2 and Satz I [9]. Q.E.D.

REMARK. [14, (4.1)] is a special case of the Corollary to Theorem 2.

\section{Extensions of global sections of coherent sheaves.}

Definition 4. Suppose $\rho$ is a natural number. A real-valued function $v$ on a complex space $X$ is said to be *-strongly $\rho$-convex at $x \in X$ if there exist a nowhere degenerate holomorphic map $\varphi$ from some open neighborhood $U$ of $x$ in $X$ to an open subset $D$ of $C^{n}$ and a real-valued $C^{2}$ function $\tilde{v}$ on $D$ such that $v=\tilde{v} \varphi$ on $U$ and at every point in $D$ the Hermitian matrix $\left(\partial^{2} \tilde{v} / \partial z_{i} \partial \bar{z}_{j}\right)_{1 \leqq i, j \leqq n}$ has at least $n-\rho+1$ positive eigenvalues.

Definition 5. Suppose $\rho$ is a natural number. An open subset $D$ of a complex space $X$ is said to be ${ }^{*}$-strongly $\rho$-concave at $x \in X$ if there is a ${ }^{*}$-strongly $\rho$-convex 
function $v$ on some open neighborhood $U$ of $x$ in $X$ such that $D \cap U=\{y \in U \mid v(y)$ $>v(x)\}$.

LEMMA 12. Suppose $\mathscr{F}$ is a coherent analytic sheaf on a reduced complex space $(X, \mathcal{O})$ of pure dimension $n$ such that $E^{n-1}(0, \mathscr{F})=\varnothing$. Suppose $1 \leqq \rho<n, x \in X$, and $D$ is an open subset of $X$ which is *-strongly $\rho$-concave at $x$. Then there exist an open neighborhood $U$ of $x$ in $X$, a subvariety $V$ of dimension $<\rho$ in $U$, and a natural number $m$ satisfying the following: If for some open neighborhood $W$ of $x$ in $U$ $f \in \Gamma(W, \mathcal{O})$ vanishes identically on $V \cap W$ and $s \in \Gamma(W \cap D, \mathscr{F})$, then $f^{m} s \mid W^{\prime} \cap D$ can be extended to an element of $\Gamma\left(W^{\prime}, \mathscr{F}\right)$ for some open neighborhood $W^{\prime}$ of $x$ in $W$.

Proof. Let $\pi:(\tilde{X}, \tilde{\mathcal{O}}) \rightarrow(X, \mathcal{O})$ be the normalization of $(X, \mathcal{O})$. Let $\tilde{\mathscr{F}}$ be the inverse image of $\mathscr{F}$ under $\pi, \mathscr{T}$ be the torsion subsheaf of $\tilde{\mathscr{F}}$, and $\mathscr{G}=\tilde{F} \mid \mathscr{T}$. Let $\pi^{-1}(x)=\left(y_{1}, \ldots, y_{k}\right)$. For every $1 \leqq i \leqq k$ there exists a sheaf-monomorphism $\alpha_{i}: \mathscr{G} \rightarrow \tilde{\mathcal{O}}^{p_{i}}$ on some open neighborhood $U_{i}$ of $y_{i}$ in $X$. By shrinking $U_{i}, 1 \leqq i \leqq k$, we can suppose that $U_{i} \cap U_{j}=\varnothing$ for $i \neq j$. There is an open neighborhood $U^{*}$ of $x$ in $X$ such that $\pi^{-1}\left(U^{*}\right) \subset \bigcup_{i=1}^{k} U_{i}$. Define a coherent analytic sheaf $\mathscr{S}$ on $\pi^{-1}\left(U^{*}\right)$ by setting $\mathscr{S}=\tilde{\mathscr{O}}^{p_{i}}$ on $\pi^{-1}\left(U^{*}\right) \cap U_{i}$ for $1 \leqq i \leqq k$. Define $\alpha: \mathscr{G} \rightarrow \mathscr{S}$ on $\pi^{-1}\left(U^{*}\right)$ by setting $\alpha=\alpha_{i}$ on $\pi^{-1}\left(U^{*}\right) \cap U_{i}$ for $1 \leqq i \leqq k$. Let $\beta: R^{0} \pi(\tilde{\mathscr{F}}) \rightarrow R^{0} \pi(\mathscr{G})$ and $\gamma$ : $R^{0} \pi(\mathscr{G}) \rightarrow R^{0} \pi(\mathscr{S})$ on $U^{*}$ be induced respectively by the quotient map $\tilde{F} \rightarrow \mathscr{G}$ and $\alpha$. Let $\lambda: \mathscr{F} \rightarrow R^{0} \pi(\tilde{F})$ be the natural map. $E^{n-1}(0, \mathscr{F})=\varnothing$ implies that $\xi=\gamma \beta \lambda: \mathscr{F} \rightarrow R^{0} \pi(\mathscr{S})$ on $U^{*}$ is injective. Let $V^{*}=E^{\rho-1}\left(\xi(\mathscr{F}), R^{0} \pi(\mathscr{S})\right)$ and let $\mathscr{I}$ be the ideal-sheaf on $U^{*}$ for $V^{*}$. By Proposition $1 \operatorname{dim} V^{*}<\rho$. Let $\mathscr{A}=\xi(\mathscr{F})$ : $\xi(\mathscr{F})_{[\rho-1] R^{0} \pi(\mathscr{S})}$. Then $E\left(\mathscr{A}, \mathcal{O} \mid U^{*}\right)=V^{*}$. Let $U$ be a relatively compact open neighborhood of $x$ in $U^{*}$. By Hilbert Nullstellensatz there is a natural number $m$ such that $\mathscr{I}^{m} \subset \mathscr{A}$ on $U$. Let $V=V^{*} \cap U$. We claim that $U, V$ and $m$ satisfy the requirements.

Suppose for some open neighborhood $W$ of $x$ in $U$ we have $f \in \Gamma(W, \mathcal{O})$ vanishing identically on $V \cap W$ and $s \in \Gamma(W \cap D, \mathscr{F})$. By Proposition 6.1, [3], for some open neighborhood $W^{\prime}$ of $x$ in $W \xi(s) \mid W^{\prime} \cap D$ can be extended to $t \in \Gamma\left(W^{\prime}, R^{0} \pi(\mathscr{S})\right)$. Let $Z=\left\{y \in W^{\prime} \mid t_{y} \notin \xi(\mathscr{F})_{y}\right\}$. $Z=E\left((\xi(\mathscr{F}): \mathcal{O} t), \mathcal{O} \mid W^{\prime}\right)$ is a subvariety in $W^{\prime}$. Since $D$ is *-strongly $\rho$-concave at $x$, every subvariety-germ of dimension $\geqq \rho$ at $x$ intersects $D$ ( $4^{0}$ of Definition 2.8 and Proposition 2.9, [3]). Hence $Z \cap D=\varnothing$ implies that $\operatorname{dim} Z_{x}<\rho$. By shrinking $W^{\prime}$, we can assume that $\operatorname{dim} Z<\rho$.

$$
t \in \Gamma\left(W^{\prime}, \xi(\mathscr{F})_{[\rho-1] R^{0} \pi(\mathscr{S})}\right) .
$$

$f^{m} t \in \Gamma\left(W^{\prime}, \xi(\mathscr{F})\right) . \xi^{-1}\left(f^{m} t\right) \in \Gamma\left(W^{\prime}, \mathscr{F}\right)$ extends $f^{m} s \mid W^{\prime} \cap D . \quad$ Q.E.D.

LEMma 13. Suppose $\mathscr{F}$ is a coherent analytic sheaf on a complex space $(X, \mathscr{H})$ of pure dimension $n$ such that $E^{n-1}(0, \mathscr{F})=\varnothing$. Suppose $1 \leqq \rho<n, x \in X$, and $D$ is an open subset of $X$ which is *-strongly $\rho$-concave at $x$. Then there exist an open neighborhood $U$ of $x$ in $X$, a subvariety $V$ of dimension $<\rho$ in $U$, and a natural number $m$ satisfying the following: If for some open neighborhood $W$ of $x$ in $U f \in \Gamma(W, \mathscr{H})$ 
vanishes identically on $V \cap W$ and $s \in \Gamma(W \cap D, \mathscr{F})$, then $f^{m} s \mid W^{\prime} \cap D$ can be extended to an element of $\Gamma\left(W^{\prime}, \mathscr{F}\right)$ for some open neighborhood $W^{\prime}$ of $x$ in $W$.

Proof. Let $\mathscr{K}$ be the subsheaf of all nilpotent elements of $\mathscr{H}$ and $\mathcal{O}=\mathscr{H} \mid \mathscr{K}$. Since the Lemma is local in nature, we can suppose that $\mathscr{K}^{k}=0$ for some natural number $k$. For $0 \leqq l \leqq k$ define $\mathscr{F}^{(l)}$ inductively as follows:

$$
\mathscr{F}^{(0)}=\mathscr{F}, \quad \text { and }, \text { for } 1 \leqq l \leqq k, \quad \mathscr{F}^{(l)}=\left(\mathscr{K}^{\mathscr{F}^{(l-1)}}\right)_{\left[n-1 \mathcal{F}^{(l-1)}\right.}
$$

As in the Proof of Lemma 5, we have the following:

$$
\mathscr{F}^{(k)}=0 ; \quad E^{n-1}\left(0, \mathscr{F}^{(l-1)} / \mathscr{F}^{(l)}\right)=\varnothing \quad \text { for } 1 \leqq l \leqq k ;
$$

and $\mathscr{G}^{(l)}=\mathscr{F}^{(l)} / \mathscr{F}^{(l+1)}, 0 \leqq l \leqq k-1$, can be regarded as a coherent analytic sheaf on the reduced complex space $(X, \mathcal{O})$. By Lemma 12 for $0 \leqq l \leqq k-1$ we have a subvariety $V_{l}$ of dimension $<\rho$ in some open neighborhood $U_{l}$ of $x$ in $X$ and a natural number $p_{l}$ satisfying the following: If for some open neighborhood $W$ of $x$ in $U_{l} f \in \Gamma(W, \mathcal{O})$ vanishes identically on $V_{l} \cap W$ and $s \in \Gamma\left(W \cap D, \mathscr{G}^{(l)}\right)$, then $f^{p_{l}} \mid W^{\prime} \cap D$ can be extended to an element of $\Gamma\left(W^{\prime}, \mathscr{G}^{(l)}\right)$ for some open neighborhood $W^{\prime}$ of $x$ in $W$.

Let $U=\bigcap \begin{gathered}k-1 \\ l=0\end{gathered} U_{l}$ and $V=\bigcup_{l=0}^{k-1}\left(V_{l} \cap U\right)$. Let $m_{l}=\sum_{i=l}^{k-1} p_{i}, \quad 0 \leqq l \leqq k-1$. By considering the exact sequences $0 \rightarrow \mathscr{F}^{(l+1)} \rightarrow \mathscr{F}^{(l)} \rightarrow \mathscr{G}^{(l)} \rightarrow 0,0 \leqq l \leqq k-1$, and by backward induction on $l$, we conclude the following for $0 \leqq l \leqq k-1:$ If $f \in \Gamma(W, \mathscr{H})$ vanishes identically on $W \cap V$ and $s \in \Gamma(W \cap D, \mathscr{F}(l)$ for some open neighborhood $W$ of $x$ in $U$, then $f^{m} s \mid W^{\prime} \cap D$ can be extended to an element of $\Gamma\left(W^{\prime}, \mathscr{F}^{(l)}\right)$ for some open neighborhood $W^{\prime}$ of $x$ in $W$. Hence $U, V$, and $m=m_{0}$ satisfy the requirements. Q.E.D.

LeMma 14. Suppose $\mathscr{F}$ is a coherent analytic sheaf on a complex space $(X, \mathscr{H})$ and $\rho$ is a natural number such that $E^{\rho}(0, \mathscr{F})=\varnothing$. Suppose $x \in X$ and $D$ is an open subset of $X$ which is *-strongly $\rho$-concave at $x$. Then there exist an open neighborhood $U$ of $x$ in $X$, a subvariety $V$ of dimension $<\rho$ in $U$, and a natural number $m$ satisfying the following: If for some open neighborhood. $W$ of $x$ in $U f \in \Gamma(W, \mathscr{H})$ vanishes identically on $W \cap V$ and $s \in \Gamma(W \cap D, \mathscr{F})$, then $f^{m} s \mid W^{\prime} \cap D$ can be extended to an element of $\Gamma\left(W^{\prime}, \mathscr{F}\right)$ for some neighborhood $W^{\prime}$ of $x$ in $W$.

Proof. Since the problem is local in nature, we can suppose that $X$ is of finite dimension $n$. If $n \leqq \rho, E^{o}(0, \mathscr{F})=\varnothing$ implies that $\mathscr{F}=0$ and what is to be proved is trivial. So we can suppose that $n>\rho$. Define $\mathscr{G}^{(k)}=0_{[k]} \mathscr{F}$ for $\rho \leqq k \leqq n$. $\mathscr{G}^{(\rho)}=0$. For $\rho<k \leqq n$ let $X_{k}=\operatorname{Supp} \mathscr{G}^{(k)} / \mathscr{G}^{(k-1)}$ and let $\mathscr{A}^{(k)}$ be the annihilator-ideal-sheaf for $\mathscr{G}^{(k)} / \mathscr{G}^{(k-1)}$. For $\rho<k \leqq n X_{k}$ is empty or of pure dimension $k, E^{k-1}\left(0, \mathscr{G}^{(k)} / \mathscr{G}^{(k-1)}\right)$ $=\varnothing$, and $\left(\mathscr{G}^{(k)} \mid \mathscr{G}^{(k-1)}\right) \mid X_{k}$ can be regarded as a coherent analytic sheaf on the complex space $\left(X_{k},\left(\mathscr{H} \mid \mathscr{A}^{(k)}\right) \mid X_{k}\right)$. By Lemma 13 , for $\rho<k \leqq n$, if $x \in X_{k}$, there exist a subvariety $V_{k}$ of dimension $<\rho$ in some open neighborhood $U_{k}$ of $x$ in $X_{k}$ and a 
natural number $p_{k}$ satisfying the following: If for some open neighborhood $W$ of $x$ in $U_{k} f \in \Gamma\left(W,\left(\mathscr{H} \mid \mathscr{A}^{(k)}\right) \mid X_{k}\right)$ vanishes identically on $W \cap V_{k}$ and

$$
s \in \Gamma\left(W \cap D, \mathscr{G}^{(k)} / \mathscr{G}^{(k-1)}\right),
$$

then $f^{p_{k S}} \mid W^{\prime} \cap D$ can be extended to an element of $\Gamma\left(W^{\prime}, \mathscr{G}^{(k)} / \mathscr{G}^{(k-1)}\right)$ for some open neighborhood $W^{\prime}$ of $x$ in $W$. For $\rho<k \leqq n$, if $x \in X_{k}$, choose an open neighborhood $\tilde{U}_{k}$ of $x$ in $X$ such that $\tilde{U}_{k} \cap X_{k}=U_{k}$; and, if $x \notin X_{k}$, let $\widetilde{U}_{k}=X, V_{k}=\varnothing$, and $p_{k}=1$.

Let $U=\bigcap_{k=\rho+1}^{n} \tilde{U}_{k}$ and $V=\bigcup_{k=\rho+1}^{n}\left(U \cap V_{k}\right)$. Set $m_{k}=\sum_{i=\rho+1}^{k} p_{i}$. By considering the exact sequences $0 \rightarrow \mathscr{G}^{(k)} \rightarrow \mathscr{G}^{(k+1)} \rightarrow \mathscr{G}^{(k+1)} / \mathscr{G}^{(k)} \rightarrow 0, \rho \leqq k \leqq n-1$, and by induction on $k$, we conclude the following for $\rho<k \leqq n$ : If for some open neighborhood $W$ of $x$ in $U f \in \Gamma(W, \mathscr{H})$ vanishes on $V \cap W$ and $s \in \Gamma\left(W \cap D, \mathscr{G}^{(k)}\right)$, then $f^{m_{k S}} \mid W^{\prime} \cap D$ can be extended to an element of $\Gamma\left(W^{\prime}, \mathscr{G}^{(k)}\right)$ for some open neighborhood $W^{\prime}$ of $x$ in $W$. The Lemma follows from $\mathscr{F}=\mathscr{G}^{(n)}$ and $m=m_{n}$. Q.E.D.

TheOREM 3 (Local EXTENSION). Suppose $\mathscr{F}$ is a coherent analytic sheaf on a complex space $(X, \mathscr{H})$ and $\rho$ is a natural number such that $\mathscr{F}=\mathscr{F}{ }^{[\rho-1]}$. Suppose $x \in X$ and $D$ is an open subset of $X$ which is *-strongly $\rho$-concave at $x$. Then the following is satisfied: If $s \in \Gamma(W \cap D, \mathscr{F})$ for some open neighborhood $W$ of $x$ in $X$, then $s \mid W^{\prime} \cap D$ can be extended to an element $t$ of $\Gamma\left(W^{\prime}, \mathscr{F}\right)$ for some open neighborhood $W^{\prime}$ of $x$ in $W$ and $t_{x}$ is uniquely determined.

Proof. Since $\mathscr{F}=\mathscr{F}^{[\rho-1]}$, by Theorem 1 , and the definition of $\mathscr{F}^{[\rho-1]}, E^{\rho}(0, \mathscr{F})$ $=\varnothing$. There exist an open neighborhood $U$ of $x$ in $X$, a subvariety $V$ of dimension $<\rho$ in $U$, and a natural number $m$ satisfying the requirements of Lemma 14. By Lemma 3 every branch of $E^{\sigma}(0, \mathscr{F})$ has dimension $>\rho$ for every nonnegative integer $\sigma$. By shrinking $U$ we can assume that there is $f \in \Gamma(U, \mathscr{H})$ such that $f$ vanishes identically on $V$ and $f$ does not vanish identically on any branch of $E^{\sigma}(0, \mathscr{F}) \cap U$ for any nonnegative integer $\sigma$. By Lemma 2 the sheaf-homomorphism $\alpha: \mathscr{F} \rightarrow \mathscr{F}$ on $U$ defined by multiplication by $f^{m}$ is injective.

Suppose $s \in \Gamma(W \cap D, \mathscr{F})$. For some open neighborhood $W^{\prime}$ of $x$ in $W \alpha(s) \mid W^{\prime}$ $\cap D=f^{m} s \mid W^{\prime} \cap D$ can be extended to an element $\tilde{t} \in \Gamma\left(W^{\prime}, \mathscr{F}\right) . Z=\left\{y \in W^{\prime} \mid z_{y}\right.$ $\left.\notin \alpha(\mathscr{F})_{y}\right\}$ is a subvariety in $W^{\prime}$. Since $D$ is *-strongly $\rho$-concave at $x$ and $Z \cap D=\varnothing$, either $x \notin Z$ or $\operatorname{dim} Z_{x}<\rho$. By shrinking $W^{\prime}$, we can assume that either $Z \cap W^{\prime}=\varnothing$ or $\operatorname{dim} Z<\rho . \tilde{t} \in \Gamma\left(W^{\prime}, \alpha(\mathscr{F})_{[\rho-1]} \mathscr{F}\right)$. $\mathscr{F}=\mathscr{F}[\rho-1]$ implies that $\alpha(\mathscr{F})_{\left[\rho-1 \mathcal{F}^{\mathscr{F}}\right.}=\alpha(\mathscr{F})$. Hence $\tilde{t} \in \Gamma\left(W^{\prime}, \alpha(\mathscr{F})\right) . t=\alpha^{-1}(\tilde{t}) \in \Gamma\left(W^{\prime}, \mathscr{F}\right)$ extends $s \mid W^{\prime} \cap D$.

Suppose for some other open neighborhood $W^{\prime \prime}$ of $x$ in $W$ there is $t^{\prime} \in \Gamma\left(W^{\prime \prime}, \mathscr{F}\right)$ extending $s \mid W^{\prime \prime} \cap D$. We are going to prove that $t_{x}^{\prime}=t_{x}$. By shrinking both $W^{\prime}$ and $W^{\prime \prime}$, we can assume that $W^{\prime}=W^{\prime \prime} . Y=\left\{y \in W^{\prime} \mid t_{y}^{\prime} \neq t_{y}\right\}$ is a subvariety in $W^{\prime}$. Since $D$ is *-strongly $\rho$-concave at $x$ and $Y \cap D=\varnothing$, either $x \notin Y$ or $t_{x}^{\prime}-t_{x} \in$ $\left(0_{[\rho-1]}\right)_{x}=0$. Q.E.D. 
Theorem 4 (Global Extension). Suppose $\rho$ is a natural number and $v$ is a *-strongly $\rho$-convex function on a complex space $X$ such that $\{x \in X \mid \lambda<v(x)<\mu\}$ is relatively compact in $X$ for any two real numbers $\lambda<\mu$. Suppose $\mathscr{F}$ is a coherent analytic sheaf on $X$ satisfying $\mathscr{F}=\mathscr{F}[\rho-1]$. Then for $\lambda \in \boldsymbol{R}$ every section of $\mathscr{F}$ on $X_{\lambda}=\{x \in X \mid v(x)>\lambda\}$ is uniquely extendible to a section of $\mathscr{F}$ on $X$.

Proof. We can assume that $X$ as a topological space is connected. Since $E^{\rho}(0, \mathscr{F})$ $=\varnothing$, we can assume that every branch of $X$ has dimension $>\rho$. Fix $\lambda_{0} \in R$ and $s \in \Gamma\left(X_{\lambda_{0}}, \mathscr{F}\right)$. We can assume that $X_{\lambda_{0}} \neq \varnothing$. Let $\Lambda=\left\{\lambda \in R \mid \lambda \leqq \lambda_{0}\right.$ and $s$ can be extended to $\left.s_{\lambda} \in \Gamma\left(X_{\lambda}, \mathscr{F}\right)\right\}$. Clearly, if $\lambda \in \Lambda$ and $\lambda<\mu$, then $\mu \in \Lambda$. We are going to prove:

$$
\begin{gathered}
\text { If } \lambda \in \Lambda \text { and } s_{\lambda}, s_{\lambda}^{\prime} \in \Gamma\left(X_{\lambda}, \mathscr{F}\right) \text { both extend } \\
s, \text { then } s_{\lambda}=s_{\lambda}^{\prime} .
\end{gathered}
$$

Suppose the contrary. Then $Z=\left\{x \in X_{\lambda} \mid\left(s_{\lambda}\right)_{x} \neq\left(s_{\lambda}^{\prime}\right)_{x}\right\}$ is a nonempty subvariety in $X_{\lambda}$. Let $Z_{0}$ be a branch of $Z$. Take $x^{*} \in Z_{0}$ and let $\lambda^{*}=v\left(x^{*}\right)$. Let $\xi=\sup \left\{v(x) \mid x \in Z_{0}\right\}$. Since $Z \cap X_{\lambda_{0}}=\varnothing, \xi$ is the supremum of $v$ on the compact set $Z_{0} \cap\left\{x \in X \mid \lambda^{*}\right.$ $\left.\leqq v(x) \leqq \lambda_{0}\right\}$. $\xi=v(y)$ for some $y \in Z_{0}$. Since $X_{\xi}$ is *-strongly $\rho$-concave at $y$ and $Z_{0} \cap X_{\xi}=\varnothing$, we have $\operatorname{dim}\left(Z_{0}\right)_{y}<\rho$. Since $Z_{0}$ is irreducible, $\operatorname{dim} Z_{0}<\rho$. Hence $\operatorname{dim} Z<\rho . s_{\lambda}-s_{\lambda}^{\prime} \in \Gamma\left(X_{\lambda}, 0_{\left[\rho-1 \mathbb{F}^{F}\right.}\right)$. (6) follows from $0_{[\rho-1]^{\mathscr{F}}}=0$.

For $\lambda \in \Lambda$ denote the unique element of $\Gamma\left(X_{\lambda}, \mathscr{F}\right)$ which extends $s$ by $s_{\lambda}$. To finish the proof, we need only prove that $\Lambda$ has no lower bound, because in that case $\Lambda=\left\{\lambda \in \boldsymbol{R} \mid \lambda \leqq \lambda_{0}\right\}$ and by (6) $s^{*} \in \Gamma(X, \mathscr{F})$ defined by $s^{*} \mid X_{\lambda}=s_{\lambda}$ for $\lambda \in \Lambda$ extends $s$. Suppose the contrary. Then $\eta=\inf \Lambda$ exists and is finite. Since $X$ is connected, this implies that $X_{\eta}$ is not closed in $X$. By Theorem 3 for every $x$ in the boundary $\partial X_{\eta}$ of $X_{\eta}$ there exists an open neighborhood $U_{x}$ of $x$ in $X$ such that $s_{\eta}$ can be extended to $t_{(x)} \in \Gamma\left(U_{x} \cup X_{\eta}, \mathscr{F}\right)$. For $x, x^{\prime} \in \partial X_{\eta}$ let $Y_{\left(x, x^{\prime}\right)}=\left\{z \in U_{x}\right.$ $\left.\cap U_{x^{\prime}} \mid\left(t_{(x)}\right)_{z} \neq\left(t_{\left(x^{\prime}\right)}\right)_{z}\right\}$. Since $0_{[\rho-1]^{F}}=\varnothing, Y_{\left(x, x^{\prime}\right)}$ is either empty or every branch of $Y_{\left(x, x^{\prime}\right)}$ has dimension $\geqq \rho$. Since $X_{\eta}$ is *-strongly $\rho$-concave at every one of its boundary points,

$$
Y_{\left(x, x^{\prime}\right)} \cap \partial X_{n}=\varnothing \quad \text { for } x, x^{\prime} \in \partial X_{n}
$$

Since $\partial X_{\eta}$ is compact we can choose $x_{1}, \ldots, x_{k} \in \partial X_{\eta}$ such that $\partial X_{\eta} \subset \bigcup_{i=1}^{k} U_{x_{i}}$. For $1 \leqq i \leqq k$ choose a relatively compact open neighborhood $W_{i}$ of $x_{i}$ in $U_{x_{i}}$ such that $\partial X_{\eta} \subset \bigcup_{i=1}^{k} W_{i}$. Let $W_{i}^{-}$be the closure of $W_{i}$ in $X, 1 \leqq i \leqq k$. (7) implies that we can choose an open neighborhood $W$ of $\partial X_{\eta}$ in $\bigcup_{i=1}^{k} W_{i}$ such that $W$ does not intersect the closed set $\bigcup_{1 \leqq i, j \leqq k, i \neq j} Y_{\left(x_{i}, x_{j}\right)} \cap W_{i}^{-} \cap W_{j}^{-}$. For some $\lambda<\eta, X_{\lambda}$ $\subset W \cup X_{\eta}$ because of Proposition 2.7 of [3]. Define $t \in \Gamma\left(X_{\lambda}, \mathscr{F}\right)$ by setting $t=s_{\left(x_{i}\right)}$ on $\left(U_{x_{i}} \cup X_{\eta}\right) \cap X_{\lambda} . t$ extends $s$, contradicting $\lambda \notin \Lambda$.

Uniqueness follows from (6). Q.E.D.

REMARKS. (i) Theorem 3 generalizes the Theorem on p. 279 of [4] and Theorem 4 generalizes Corollary 5.2 of [4] because of Theorem 4.3 of [4]. Theorems 3 and 4 
here have the advantage that, if $\mathscr{F}$ does not satisfy $\mathscr{F}=\mathscr{F}^{[\rho-1]}$, we can always construct the coherent analytic sheaf $\mathscr{G}=\left(\mathscr{F} / 0_{[\rho]} \mathscr{F}\right)^{[\rho-1]}$ which satisfies $\mathscr{G}=\mathscr{G}^{[\rho-1]}$.

(ii) Suppose $\mathscr{F}$ is a coherent analytic sheaf on a complex space $(X, \mathscr{H})$ and $x \in X$. The condition $\mathscr{F}_{x}=\left(\mathscr{F}^{[0]}\right)_{x}$ is equivalent to the condition codh $\mathscr{F}_{x} \geqq 2$. It can be proved in the following way: If $\mathscr{F}_{x}=\left(\mathscr{F}^{[0]}\right)_{x}$, then $E^{0}(0, \mathscr{F})=\varnothing$ and by Lemmas 2 and 3 we can find $f \in \Gamma(U, \mathscr{H})$ for some open neighborhood $U$ of $x$ in $X$ such that $f_{x}$ is not a unit of $\mathscr{H}_{x}$ and $f_{x}$ is not a zero-divisor for $\mathscr{F}_{x}$. By shrinking $U$, we can assume that $f_{y}$ is not a zero-divisor for $\mathscr{F}_{y}$ for $y \in U$. Suppose $x \in E^{0}(f \mathscr{F}, \mathscr{F} \mid U)$. By shrinking $U$, we can find $g \in \Gamma(U, \mathscr{F})$ such that $g_{y} \in(f \mathscr{F})_{y}$ for $y \in U-\{x\}$ and $g_{x} \notin(f \mathscr{F})_{x}$. Then $h \in \Gamma\left(U, \mathscr{F}^{[0]}\right)$ defined by $g_{y}=f_{y} h_{y}$ for $y \in U-\{x\}$ does not satisfy $h_{x} \in \mathscr{F}_{x}$. This is a contradiction. Hence $x \notin E^{0}(f \mathscr{F}, \mathscr{F} \mid U)$. By Lemmas 2 and 3 we can find $s \in \mathscr{H}_{x}$ which vanishes at $x$ and is not a zero-divisor for $(\mathscr{F} / f \mathscr{F})_{x} \cdot \operatorname{codh} \mathscr{F}_{x}$ $\geqq 2$. On the other hand codh $\mathscr{F}_{x} \geqq 2$ implies $\mathscr{F}_{x}=\left(\mathscr{F}^{[0]}\right)_{x}$ by Korollar zu Satz III, [9].

The equivalence of $\mathscr{F}_{x}=(\mathscr{F}[0])_{x}$ and $\operatorname{codh} \mathscr{F}_{x} \geqq 2$ is also a consequence of $[14,(1.1)]$. However, the proof presented here is more conceptual than the proof in [14].

(iii) In the case of Stein spaces we have the following stronger version of Theorem 4 which generalizes Theorem 5.4 of [4]:

Suppose $\mathscr{F}$ is a coherent analytic sheaf on a Stein space $X$ such that $\mathscr{F}=\mathscr{F}^{[0]}$. Suppose $K$ is a compact subset of $X$ such that, if $A$ is a branch of $E^{\sigma}(0, \mathscr{F})$ for any $\sigma \geqq 2$, then $A-K$ is irreducible. Then for every open neighborhood $U$ of $K$ in $X$ every element of $\Gamma(U-K, \mathscr{F})$ can be extended uniquely to an element of $\Gamma(U, \mathscr{F})$.

It can be proved in the following way: Suppose $s \in \Gamma(U-K, \mathscr{F})$. Since $H^{1}(X, \mathscr{F})$ $=0$, from the Mayer-Vietoris sequence of $\mathscr{F}$ on $X=(X-K) \cup U$ (p. 236, [2]) we conclude that for some $f \in \Gamma(X-K, \mathscr{F})$ and $g \in \Gamma(U, \mathscr{F}) f-g=s$ on $U-K$. From Theorem 4 we can find $\tilde{f} \in(X, \mathscr{F})$ which agrees with $f$ outside some compact subset of $X$. Since $E^{\sigma}(0, \mathscr{F})=\varnothing$ for $\sigma \leqq 1$ and $A-K$ is irreducible for any branch $A$ of $E^{\sigma}(0, \mathscr{F})$ with $\sigma \geqq 2, f$ agrees with $\tilde{f}$ on $X-K$. $(\tilde{f} \mid U)-g$ extends $s$. The extension is clearly unique, because $E^{0}(0, \mathscr{F})=\varnothing$.

In view of the equivalence of $\mathscr{F}_{x}=\left(\mathscr{F}^{[0]}\right)_{x}$ and $\operatorname{codh} \mathscr{F}_{x} \geqq 2$, in the above proof we can use Theorem 15 of [2] instead of Theorem 4 . So (8) can be proved also by the finiteness theorems of pseudoconvex spaces in [2].

(8) generalizes Theorem 5.4 of [4] because of the following:

Suppose $K$ is a closed subset of an irreducible complex space $X$ and $U$ is an open neighborhood of $K$ in $X$ such that for every branch $A$ of $U A-K$ is irreducible. Then $X-K$ is irreducible. 
Let $R$ be the set of all regular points of $X$. To prove (9), we need only show that $R-K$ is connected. Suppose $R \cap U=\bigcup_{i \in I} R_{i}$ is the decomposition into topological components. Then $R_{i}-K$ is connected for $i \in I$. The restriction map $\Gamma(R \cap U, C) \rightarrow$ $\Gamma(R \cap(U-K), C)$ is an isomorphism. From the following portion of the Mayer-Vietoris sequence of the constant sheaf $C$ on $R=(R \cap U) \cup(R-K)$ : $0 \rightarrow \Gamma(R, C) \rightarrow \Gamma(R-K, C) \oplus \Gamma(R \cap U, C) \rightarrow \Gamma(R \cap(U-K), C)$, we conclude that the restriction map $\Gamma(R, C) \rightarrow \Gamma(R-K, C)$ is an isomorphism. $R-K$ is connected.

\section{REFERENCES}

1. A. Andreotti, Théorèmes de dépendance algébrique sur les espaces complexes pseudoconcaves, Bull. Soc. Math. France 91 (1963), 1-38.

2. A. Andreotti and H. Grauert, Théorèmes de finitude pour la cohomologie des espaces complexes, Bull: Soc. Math. France 90 (1962), 193-259.

3. H. Fujimoto, On the continuation of analytic sets, J. Math. Soc. Japan 18 (1966), 51-85.

4. - The continuation of sections of torsion-free coherent analytic sheaves, Nagoya Math. J. 31 (1968), 279-294.

5. H. Grauert, Ein Theorem der analytischen Garbentheorie und die Modulräume komplexer Strukturen, Inst. Hautes Études Sci. Publ. Math. No. 5, 1960.

6. H. Grauert and R. Remmert, Bilder und Urbilder analytischer Garben, Ann. of Math. 68 (1958), 393-443.

7. R. C. Gunning and H. Rossi, Analytic functions of several complex variables, PrenticeHall, Englewood Cliffs, N. J., 1965.

8. M. Nagata, Local rings, Interscience, New York, 1962.

9. G. Scheja, Riemannsche Hebbarkeitssätze für Cohomologie Klassen, Math. Ann. 144 (1961), 345-360.

10. — Fortsetzungssätze der komplex-analytischen Cohomologie und ihre algebraische Charakterisierung, Math. Ann. 157 (1964), 75-94.

11. J.-P. Serre, Prolongement de faisceaux analytiques cohérents, Ann. Inst. Fourier (Grenoble) 16 (1966), 363-374.

12. Y.-T. Siu, Noether-Lasker decomposition of coherent analytic subsheaves, Trans. Amer. Math. Soc. 135 (1969), 375-384.

13. W. Thimm, Lückengarben von kohärenten analytischen Modulgarben, Math. Ann. 148 (1962), 372-394.

14. G. Trautmann, Ein Kontinuitätssatz für die Fortsetzung kohärenter analytischen Garben, Arch. Math. (Basel) 18 (1967), 188-196.

UNIVERSITY OF NOTRE DAME, Notre Dame, Indiana 